\title{
Proposal of Mode Selection Criterian for Advanced KDEMO Gyrotron
}

\author{
Ashwini Sawant ${ }^{1}$, Mun Seok Choe $^{2}$, and EunMi Choi ${ }^{2, a}$ \\ ${ }^{1}$ Department of Electrical and Computer Engineering, UNIST, UIsan, Republic of Korea. \\ ${ }^{2}$ School of Natural Science, UNIST, UIsan, Republic of Korea.
}

\begin{abstract}
We proposed the design of a $255 \mathrm{GHz}$ advanced gyrotron for Korea demonstration power plant (KDEMO) operation. Mode selection criterian for this DEMO gyrotron (output power $>1 \mathrm{MW}$ ) is discussed and a conventional cavity is designed for selected $\mathrm{TE}_{55,13}$ mode. The designed cavity also excites the $\mathrm{TE}_{37,9}$ mode at $170 \mathrm{GHz}$ frequency. Beam wave interaction at both the frequencies are also analysed in our study.
\end{abstract}

\section{Introduction}

Gyrotron oscillators delievering MW's of power at millimeter wave regime are considered as a RF source for Electron cyclotron resonance heating $(\mathrm{ECRH})$ and current drive (CD) in nuclear fusion reactors. DEMO [1] which is a futuristic ambitious step in thermonuclear fusion reactor field beyond ITER requires an advanced gyrotron for ECRH and ECCD [2]. The gyrotron is required to deliver MW of power along with the frequency tuning capability [3]. Conventional cavity gyrotron and coaxial cavity gyrotron are under investigation for $255 \mathrm{GHz}$ operation. Here we have presented the mode selection criteria and initial design for a conventional type gyrotron cavity.

\section{KDEMO Gyrotron Design}

\subsection{Mode Selection}

To deliver high power of MW order at high frequency, a higher order mode must be selected. Apart from the conventional technique of mode selection, frequency tuning and output power criteria also play a major role in selection of the mode. Multi-frequency operation is only possible when all the operating frequencies are supported by the output window and the quasi-optical launcher. Output window is supported by multiple frequencies when these frequencies are its harmonics. In our gyrotron design, 170 $\mathrm{GHz}$ and $255 \mathrm{GHz}$ are chosen as operating frequencies as these two frequencies are harmonics of $28.33 \mathrm{GHz}$ and support ITER and DEMO operation respectively. For a feasible operation of quasi-optical launcher the selected modes must have the same caustic radius. $\mathrm{TE}_{55,13}$ and $\mathrm{TE}_{37,9}$ are the two modes that have almost same caustic radius and resonate at $255 \mathrm{GHz}$ and $171.7 \mathrm{GHz}$ respectively inside a conventional cavity of $20.78 \mathrm{~mm}$ radius. Fig. 1 shows the density of the mode spectrum around $255 \mathrm{GHz}$ and relative couplings of the modes at optimum beam radius of $10.68 \mathrm{~mm}$.

\footnotetext{
ae-mail: emchoi@unist.ac.kr
}

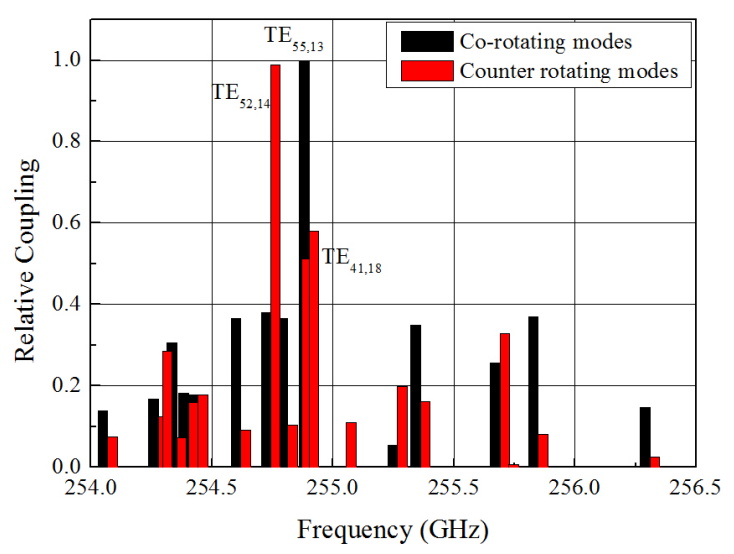

Figure 1. Relative coupling of the $\mathrm{TE}_{55,13}$ and its neighboring modes for beam radius of $10.68 \mathrm{~mm}$.

\subsection{Cavity Design}

Interaction of the beam and field takes place inside an open ended cavity in gyrotron. conventional cavity [4] and coaxial cavity [5] are the two popular type of cavities inherited for gyrotron design. In case of High Power Gyrotron ex. ITER and DEMO Gyrotron, which generally works on higher order mode to generate the MW's of power, coaxial type cavity has an advantage of reduced mode competition over conventional type of cavity. Gyrotron with coaxial type cavity can produce comparatively higher power but at the cost of more sophisticated design. We are analyzing the both type of cavities for 255 $\mathrm{GHz}$ Gyrotron but here we will just focus on conventional type of gyrotron cavity. A conventional gyrotron cavity is designed with an in house developed code "UNIST gyrotron design Tool (UGDT)" as shown in Fig. 2 which excites $\mathrm{TE}_{55,13}$ mode at $254.99 \mathrm{GHz}$ with a quality factor of 1186 . We also performed the cold test of the cav- 


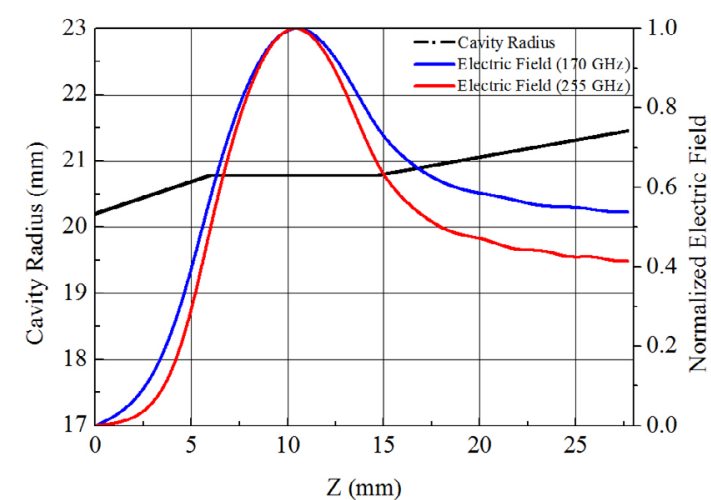

Figure 2. Normalized electric field along the cavity length.

ity at around $170 \mathrm{GHz}$ and we found that $\mathrm{TE}_{37,9}$ mode also resonates inside our designed cavity at $171.7 \mathrm{GHz}$ but with a relatively smaller quality value of 560 as shown in Fig. 2. The geometrical parameters of this cavity are tabulated in Table 1.The beam wave interaction analysis is also carried out using time independent self consistent UGDT code. The beam wave interaction analysis shows maximum power of $1.12 \mathrm{MW}$ at $10.16 \mathrm{~T}$ magnetic field for $\mathrm{TE}_{55,13}$ as shown in Fig. 3 for a high quality electron beam as tabulated in Table 2. It also predicts a maximum power of $1.5 \mathrm{MW}$ at 6.35 magnetic field for $\mathrm{TE}_{37,9}$ mode as shown in Fig. 3.

Table 1. Cavity Geometry

\begin{tabular}{|l|l|}
\hline Design Parameter & Value \\
\hline Input Taper Radius $\left(\mathrm{R}_{1}\right)$ & $20.2 \mathrm{~mm}$ \\
\hline Cavity Radius $\left(\mathrm{R}_{0}\right)$ & $20.78 \mathrm{~mm}$ \\
\hline Output Taper Radius $\left(\mathrm{R}_{3}\right)$ & $21.5 \mathrm{~mm}$ \\
\hline Input Taper Length $\left(\mathrm{L}_{1}\right)$ & $6 \mathrm{~mm}$ \\
\hline Mid-section Length $\left(\mathrm{L}_{2}\right)$ & $8.7 \mathrm{~mm}$ \\
\hline Output Taper Length $\left(\mathrm{L}_{3}\right)$ & $13 \mathrm{~mm}$ \\
\hline
\end{tabular}

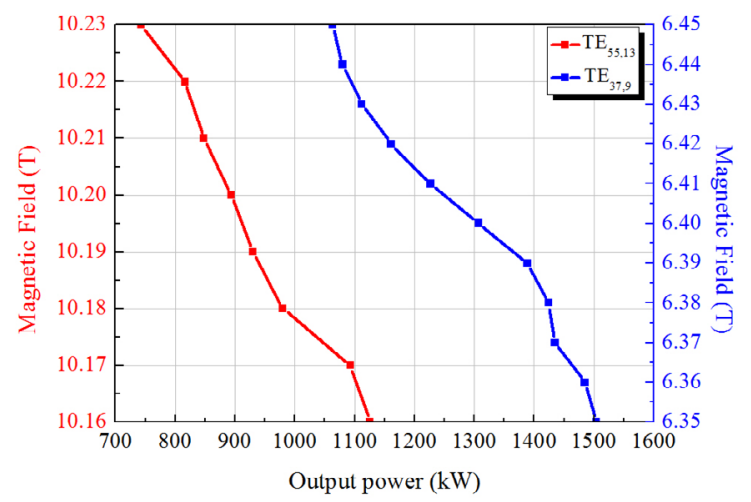

Figure 3. Magnetic Field Vs Output Power for both modes.
Table 2. Gyrotron Design Parameters

\begin{tabular}{|l|l|}
\hline Design Parameter & Value \\
\hline Beam Voltage $\left(\mathrm{U}_{\mathrm{b}}\right)$ & $80 \mathrm{kV}$ \\
\hline Beam Current $\left(\mathrm{I}_{\mathrm{b}}\right)$ & $40 \mathrm{~A}$ \\
\hline Alpha $(\alpha)$ & 1.3 \\
\hline Magnetic Field $\left(\mathrm{B}_{0}\right)$ & $10.2 \mathrm{~T}$ \\
\hline Beam Radius $\left(\mathrm{R}_{\mathrm{b}}\right)$ & $10.68 \mathrm{~mm}$ \\
\hline
\end{tabular}

\section{Conclusion}

A rigorous analysis of mode selection for KDEMO gyrotron is carried out to generate MW of power at multi-frequencies by using a UNIST gyrotron design Tool (UGDT). The cavity design and its interaction with the beam is also analyzed using UGDT. The design of the quasi-optical launcher and RF window is still under investigation.

\section{References}

[1] K. Sakamoto, K. Takahashi, A. Kasugai, R. Minami, N. Kobayashi, S. Nishio, M. Sato, K. Tobita, Fusion Engineering and Design 81, 1263 (2006)

[2] J. Jelonnek, K. Avramidis, J. Franck, G. Gantenbein, K. Hesch, J. Jin, P. Kalaria, A. Malygin, I.G. Pagonakis, T. Rzesnicki et al., in Infrared, Millimeter, and Terahertz waves (IRMMW-THz), 2014 39th International Conference on (2014), pp. 1-3

[3] P.C. Kalaria, K.A. Avramidis, J. Franck, S. Illy, I.G. Pagonakis, M. Thumm, J. Jelonnek, in Vacuum Electronics Conference (IVEC), 2015 IEEE International (2015), pp. 1-2.

[4] A. Sawant, S.G. Kim, M.C. Lin, J.H. Kim, Y. Hong, J. So, E. Choi, IEEE Transactions on Plasma Science 42, 3989 (2014)

[5] B. Piosczyk, A. Arnold, G. Dammertz, O. Dumbrajs, M. Kuntze, M.K. Thumm, IEEE Transactions on Plasma Science 30, 819 (2002) 